\title{
2 Aufgaben von Primarschulen in herausfordernden Lagen
}

Welche Aufgaben Schulen zu erfüllen haben, hängt von gesellschaftlichen Ansprüchen und schulspezifischen Kontextbedingungen ab. Je nach theoretischer Perspektive werden die Aufgaben der Schule unterschiedlich verstanden und gewichtet. Als gemeinsamer Nenner dieser Perspektiven kann die Aufgabe der Förderung und der Selektion als Kerngeschäft der Primarschule gesehen werden. Verschiedene Aufgaben und Funktionen von Schulen mit Fokus auf der Förder- und Selektionsaufgabe werden im folgenden Kapitel theoretisch reflektiert. Dies dient der Kontextualisierung der Arbeit mit empirischem Fokus auf der Rekonstruktion von Orientierungen der Förderung und Selektion. Als weitere Grundlage ist die Arbeit angesiedelt im Forschungskontext von Schulen in herausfordernden Lagen. Dieser Begrifflichkeit und Einordnung widmet sich das nachfolgende Unterkapitel.

\subsection{Aufgaben und Funktionen von Primarschulen}

Welche Aufgaben die Schule erfüllen soll und welche Funktionen sie hat, hängt vom theoretischen Paradigma ab, das der Vorstellung von Bildung und Schule zugrunde liegt. Grundsätzlich hat die Schule eine Verpflichtung einerseits gegenüber den Individuen und ihrer Entwicklung, andererseits gegenüber der Gesellschaft. Aus einer anthropologischen Perspektive, wie dies unter anderem von Hentig (2003) formuliert, wird Schule insbesondere als ,Polis ${ }^{`}$ gedacht mit Fokus auf der Stärkung des Individuums in seinem Lebensraum und auf der Schule als Erfahrungsraum, welcher auf gesellschaftliche Partizipation vorbereitet (vgl. Hentig, 2003, S. 190-191). Dieser Ansatz kann als Gegenentwurf zu dem in vielen Gesellschaften als Reaktion auf die OECDVergleichsstudien dominierenden Leistungsoutput-Ansatz gesehen werden. Die Aufforderung, Schule neu zu denken, die gemäß dem Autor im Anschluss an diese Studien wieder an Aktualität gewonnen hat, basiert auf einem ganzheitlich auf den Mensch gerichteten Ansatz (vgl. Hentig, 2003, S. V26-V28).

Im Unterschied dazu wird die Schule aus systemtheoretischer Perspektive als Subsystem der Gesellschaft und als Sozialisationsinstanz neben anderen verstanden. Mit Blick auf die Frage, welche Aufgaben Schulen erfüllen sollen, bietet Luhmann ${ }^{4}$ in Das Erziehungssystem der Gesellschaft (2002) mögliche systemtheoretische Antwor-

4 Einige der hier zitierten Standardwerke von Luhmann (†1998), wie Organisation und Entscheidung (2006), Das Erziehungssystem der Gesellschaft (2002) und auch Einführung in die Systemtheorie (Neuauflage 2011), wurden posthum herausgegeben; die Texte basieren auf Schriften, welche Luhmann mehrheitlich in den 1980er Jahren verfasste.

(C) Der/die Autor(en) 2019

C. Kamm, Konzeptionen von Förderung,

Selektion und Gerechtigkeit, Rekonstruktive Bildungsforschung 23,

https://doi.org/10.1007/978-3-658-25782-8_2 
ten. Sozialisation in schulischen Organisationen beinhaltet gemäß Luhmann (2002, S. 51) per se eine Differenzierung in der Bereitstellung verschiedener Leistungsschienen. Dies dient dazu, Sozialisationsprozesse auf die permanente Unsicherheit des menschlichen Handelns ausrichten zu können (ebd., S. 50). Differenzierung und Selektion sind im Bildungssystem eingeschrieben und somit grundlegende Aufgaben von schulischen Organisationen. Gleichzeitig grenzt sich die Organisation gegenüber der Umwelt, beispielsweise den Familien, ab, indem sie sich bemüht, ungleiche Voraussetzungen durch Homogenisierung im Schuleintritt auszugleichen (ebd., S. 127). Die Aufgabe der Sozialisation ist eng mit dem Thema Chancengerechtigkeit verknüpft, da gemäß Luhmann (2002, S. 127-128) die Homogenisierung der Eintrittspopulation zu einer markanten Ausdifferenzierung des Erziehungssystems führen kann, wobei Unterschiede nun systemintern produziert werden.

Innerhalb der Schule führen diese beiden unterschiedlichen Vorstellungen von Bildung zu Herausforderungen. Einerseits soll durch individualisierte Förderung auf (benachteiligte) Voraussetzungen eingegangen werden, gleichzeitig ist Selektion aufgrund systembedingter Ergebnisungleichheit unumgänglich. Organisationen des Bildungssystems sind mit der Verteilung von Chancen beauftragt. Dadurch sind sie nicht nur an der Bearbeitung, sondern auch am Erhalt sozialer Differenz in der Reproduktion gesellschaftlicher Sozialstrukturen beteiligt (Graf \& Graf, 2008). Im bildungspolitischen Diskurs wird das Erfordernis der Selektion gerne auch mit dem Argument verteidigt, dass dann, wenn die Volksschule nicht selektioniere, dies andere Instanzen der Aufnahmesysteme übernähmen, wie es beispielsweise der Dachverband Lehrer/-innen der Schweiz (LCH) im Positionspapier zur Kompetenzerreichung im Lehrplan 21 formuliert hat (Dachverband Lehrerinnen und Lehrer Schweiz, 2014). Diese Begründung wird auch in wissenschaftlichen Studien vertreten, wobei es als ein Verlust der Berechtigungsfunktion der Schule gilt, wenn die Selektionsfunktion anderen Systemen überlassen wird, da sich dies letztendlich kontraproduktiv auf die Korrektur statusbezogener Reproduktionsmuster auswirken würde (vgl. Terhart, 2001).

Die Einbettung der Schule und ihrer Akteure in ihren Systemzusammenhang beschreibt Fend (1981) in seiner Theorie der Schule. Fend unterscheidet zwischen verschiedenen ,gesellschaftlichen Funktionen des Bildungswesens“ (Fend, 1981, S. 1516), welche das Verhältnis des Bildungssystems zur Gesellschaft und die daraus entstehenden Erwartungen an Schule reflektieren. Aufgaben von Schule werden von verschiedenen Autoren diskutiert, wobei als gemeinsamer Nenner insbesondere Qualifikation, Selektion und Sozialisation/Legitimation gesehen werden kann (u. a. Fend, 1981; Sandfuchs \& Melzer, 2001; Wiater, 2009). Die Qualifikationsfunktion zielt auf die Qualifizierung der Schüler/-innen gemäß den Anforderungen des Beschäftigungssystems, wobei die Herausforderungen sich aus der wirtschaftlichen Situation und der Passung zwischen schulischen Qualifikationen und arbeitsmarktlichen Erfordernissen ergeben (Fend, 1981, S. 28). Die Selektionsfunktion wirkt durch das Erteilen von Zeugnissen und Zugangsberechtigungen auf die Reproduktion der Sozialstruktur und 
das berufliche Positionssystem. Fend (1981) verweist dabei auf den „Zwang zur frühen Selektion und ein fehlendes Bemühen zum Ausgleich von familiär mitgebrachten Lerndefiziten“ (ebd., S. 39) als zentrale Herausforderung. Die Legitimationsfunktion schließlich zielt nach Fend (1981, S. 46) auf die Akzeptanz der Zuordnung verschiedener Leistungsdispositionen zu formellen Belohnungen. Erzeugt die Vermittlung von Normen und Wertorientierungen eine systemstabilisierende Wirkung, so besteht die Herausforderung für die Bildungseinrichtungen aber auch darin, sich kritisch mit deren Inhalten auseinanderzusetzen (Fend, 1981, S. 49). Auf der Basis dieser Funktionen wird Selektion im heutigen Bildungssystem normativ dann als legitim erachtet, wenn sie dem meritokratischen Anspruch, dass die Unterschiede auf Leistungsdifferenzen basieren, entspricht; als illegitim gilt sie, wenn sie kategorial erfolgt, also leistungsfremd ist (Hormel, 2010, S. 176). Verschiedene Studien zeigen allerdings, dass Selektionsentscheide über differenzierte Schultypen gesellschaftliche Ungleichheiten transformieren und verfestigen, und dies auch entlang leistungsferner Kategorien wie z. B. Herkunftsmerkmale wie Migrationsstatus oder Sozialschicht (Becker \& Schulze, 2013; Sturm, 2013).

Neben dieser Reproduktionsfunktion hat die Schule aber auch die Aufgabe der ganzheitlichen Persönlichkeitsentwicklung. Sie gerät in der systemtheoretischen Betrachtungsweise und in der Formulierung von Aufgaben und Funktionen oftmals in den Hintergrund. In einem stärker auf dem Humboldt'schen Verständnis von Schule basierenden Bildungsbegriff als Menschenbildung steht die Persönlichkeitsentwicklung des Kindes bzw. Jugendlichen im Zentrum. Die Begriffe ,Qualifikation“ oder ,Förderung' werden hier umfassend und unabhängig von späterem Nutzen verstanden (Koller, 2014). Ihre Aufgabe kann daher unterschiedliche Konnotationen beinhalten, was sich auf den Umgang mit Bildungsungleichheiten auswirkt.

In beiden Ansätzen, sowohl in der systemischen als auch in der humanistischen Logik, tritt der gesellschaftliche Reproduktionsauftrag der Schule in ein Spannungsverhältnis mit der Aufgabe, die individuelle Persönlichkeitsentwicklung zu ermöglichen. Dabei wird die Bearbeitung von Ungleichheit oft als pädagogisches Paradigma der staatlich aufgezwungenen Selektion gegenübergestellt (u. a. Luhmann \& Lenzen, 2002, S. 62). Diese Relation zwischen pädagogischem Paradigma der Erziehung und systemisch aufgezwungener Selektion wird als wechselseitiges Bedingungsgefüge angesehen, welches sowohl in der Kultur der Lehrpersonen als auch in jener der Schülerschaft etabliert ist (ebd., S. 63). Das Erziehungssystem bemüht sich durch gleiche Angebote und Hilfestellungen um Bildungsgerechtigkeit, die Ergebnisungleichheit ist dem System jedoch eingeschrieben (ebd., S. 129). Die pädagogische Prämisse der Lehrperson mit Blick auf Erziehung reflektiert Breidenstein (2012) in seiner Studie Das Theorem der Selektionsfunktion kritisch. Er zeigt auf, dass Selektion keineswegs nur Vollzug eines äußeren Auftrags ist, sondern systemintern zur Legitimation des Berufsauftrages des Pädagogen dient. Dies ergebe sich daraus, dass Abnehmersysteme eigene Selektionskriterien haben, während die selektionsrelevanten Entscheidungen dazu dienen, den Verbleib und die Zuteilung innerhalb des Systems zu gewährleisten 
(Breidenstein, 2012, S. 14). Dass die Differenzkonstruktion für die pädagogische Arbeit auch strukturierend und sinnstiftend wirken kann, haben auch andere Autoren konstatiert (bspw. Budde, 2015).

Dieses in das System, aber auch in die Vorstellung von Bildung verschiedener Schulakteure eingeschriebene Spannungsfeld zwischen gesellschaftlicher Reproduktionsfunktion durch Selektion und Persönlichkeitsentwicklung durch Förderung scheint nicht leicht auflösbar. Die duale Aufteilung von Selektion als äußerer Auftrag und Förderung als pädagogische Prämisse ist zu kurz gefasst. Empirische Ergebnisse wie beispielsweise die Studie von Breidenstein (2012) zeigen, dass Selektion auch schulsystemintern sowie professionserleichternd wirken kann. Äußerlich wird sie zwar kritisiert, gleichzeitig kann sie jedoch als gewollter Bestandteil der Aufgaben in der Schule angesehen werden. Auch Förderung kann je nach Perspektive unterschiedlich konnotiert sein. Wird sie als Differenzierungsmerkmal im inklusiven Unterricht eingesetzt, dient sie unter Umständen auch als Selektionsinstrument.

Die Frage, unter welchen Bedingungen Förderung und Selektion von den Schulakteuren als gerecht wahrgenommen wird, ist empirisch zu prüfen. Besondere Beachtung findet dies im Forschungsfeld von Schulen in herausfordernden Lagen.

\subsection{Schulen in herausfordernden Lagen}

Die Arbeit ist angesiedelt im Forschungskontext von Schulen in herausfordernden Lagen. Von ihnen wird insbesondere im Zusammenhang mit räumlichen Segregationsprozessen in urbanen Gebieten gesprochen. Urbane Segregation meint die ungleiche Verteilung bestimmter Bevölkerungsgruppen über ein Stadtgebiet (Häußermann et al., 2008). Den Begriff der Segregation prägte die englischsprachige, insbesondere die amerikanische Forschung, welche in den 1960er Jahren ethnische Ungleichverteilungen von weißen und nicht weißen Bevölkerungsschichten in Großstädten im Zusammenhang mit dem Zugang zu Bildung und Arbeit untersuchten. In Abgrenzung von der residentiellen Segregation bezieht sich dies auf das städtische Phänomen, dass soziale Ungleichheiten im städtischen Raum abgebildet sind (Mafaalani, Kurtenbach \& Strohmeier, 2015, S. 9). Soziologische Studien städtischer Segregation untersuchen insbesondere die Ungleichverteilung sozialer, ethnischer und demographischer Bevölkerungsschichten (ebd.).

Studien, welche Segregation in deutschen Städten untersuchen, sprechen von einer Überlagerung der Dimensionen. So wohnen in sozial deprivierten Quartieren beispielsweise überproportional viele Migrantinnen und Migranten mit geringen oder nicht anerkannten Bildungstiteln, wobei diese Gruppe im Vergleich zur Durchschnittsbevölkerung eine hohe Kinderanzahl besitzt (Strohmeier, 2008). Diese Befunde können - wenngleich aufgrund der Größe der Städte in geringerem Ausmaß - auch auf die Schweiz übertragen werden. Im Unterschied zu soziologisch ausgerichteten Studien, welche die Ursachen urbaner Segregation untersuchen, fassen erziehungswissenschaft- 
liche Studien Segregation als Bedingung oder Herausforderung schulischer Arbeit auf (Fölker, Hertel \& Pfaff, 2015, S. 17). Angloamerikanische Forschungen, zu deren Ziel „,improving schools in challenging circumstances“ gehört, definieren beispielsweise sinnvolle Strategien für das Lern- und Unterstützungsangebot an die Schüler/-innen (Harris et al., 2006; Muijs, Harris, Chapman, Stoll \& Russ, 2004). Ähnlich fokussiert die Forschung unter dem Stichwort „failing schools“ (u. a. Quesel, Husfeldt, Landwehr \& Steiner, 2013) das Zusammenwirken von internen und externen Faktoren problematischer Konstellationen und zielt so ebenfalls auf die Prozessqualität auf Unterrichtsebene. Dies wird von der deutschsprachigen Forschung beispielsweise im Sammelband Gute Schulen in schlechter Gesellschaft (Lohfeld, 2008) aufgegriffen und von verschiedenen Autoren diskutiert. Solche Studien im Kontext der internationalen Schuleffektivitätsforschung folgen dabei insbesondere einer Logik von Input (Herkunftsmerkmale der Schüler/-innen) und Output (Herausforderungen für Kompetenzerwerb und Schulentwicklung) (van Ackeren, 2008). Fölker et al. (2015, S. 21) bemängeln jedoch das Fehlen einer stärker strukturellen Betrachtung des Phänomens. Dies wäre beispielsweise in einer Analyseeinstellung denkbar, welche auf die Konstruktion der Kategorie sozialer Herkunft von Schülerinnen und Schülern und deren Klassifikation in Förderungs- und Selektionsprozessen achtet.

Neuere Sammelbände zu Schulen in ,schwieriger “ Lage (Bremm et al., 2016) oder Brennpunkt(-)Schule (Fölker et al., 2015) versuchen, Thematiken lokalräumlicher Dynamiken der Bildungssegregation sowie der Konstruktionsleistungen von Schulen mit aufzunehmen. Die Fremd- und Selbstetikettierung als „schwierige“ Lage ist im bildungspolitischen und im wissenschaftlichen Diskurs gemäß Bremm et al. (2016) weit verbreitet. Dabei diskutieren die Autoren neben scheinbar objektiven Zugehörigkeiten auch gerade implizite Zuschreibungen auf Schulebene, welche als Multiplikator von Benachteiligungen wirken können (Bremm et al., 2016). Die Schule selbst kann durch Re-Identifikation ihrer sozialräumlich benachteiligten Lage an deren Konstruktion mitbeteiligt sein (Bremm et al., 2016, S. 326). Hummrich (2015, S. 174-175) begreift die Schule als aktiv konstruierend; sie geht von der Annahme aus, dass sich die Schule selbst ,als etwas“ symbolisch entwirft, was in Differenz zu den Strukturbedingungen der Umwelt stehen kann. Im Schweizer Kontext beschäftigt sich u. a. eine Studie zu Selektionskulturen in einem segregierten Stadtteil in Bern mit dem Thema (Oester, Brunner \& Fiechter, 2015). Sie kommt auf der Grundlage einer qualitativen Untersuchung zu dem Ergebnis, dass ,,weder der Ausländeranteil allein noch das Milieu, aus dem die Kinder stammen, für die Sekundarschulübertrittsraten und damit den messbaren Output der Schülerleistungen verantwortlich sind“ (Oester et al., 2015, S. 99). Vielmehr sei die Kombination einer marktorientierten Bildungspolitik mit der institutionellen Geschichte der Schule der Grund für unterschiedliche Selektionskulturen im Rahmen der Schule (Oester et al., 2015).

In dieser Arbeit wird an diese bildungspolitische Diskussion zu Schulen in ,schwierigen Lagen` angeknüpft. Es wird der Begriff der angloamerikanischen ,chal- 
lenging circumstances' verwendet, in der Annahme, dass das Prädikat schwierig respektive herausfordernd eine äußere sowie innere Konstruktionsleistung darstellt. Diese Wahl begründet sich darin, dass von einem konstruktivistischen Verständnis der Adressierung der Schule mit ihrer Lage ausgegangen wird. So werden Schulen nicht als passive Akteure ihres Kontextes gesehen, sondern es wird davon ausgegangen, dass sie sich aktiv zu ihrem Kontext in Beziehung setzen, diesen reflektieren und auch mitbedingen. Es wird an das von Fölker et al. (2015) hervorgehobene Forschungsdesiderat angeknüpft, die Rolle der Schule und ihrer Konstruktionsleistung des sozialen Kontexts im Hinblick auf Förderung und Selektion mitzudenken. Weiter wird die Lage als ,herausfordernd', nicht als ,schwierig', als ,schlechte Gesellschaft' oder als ,failing" verstanden, da nicht die Wertung der Lage, sondern der Umgang mit ihr im Zentrum steht. ,Herausfordernd' ist dabei wertneutral zu verstehen und kann auch weitere wahrgenommene Herausforderungen im Zusammenhang mit der Schüler- und Elternschaft umfassen, beispielsweise sich wandelnde Ansprüche. Im Forschungskontext sind dabei nicht nur segregierte Quartierslagen und die aktive Auseinandersetzung mit ihnen ein Thema, sondern auch der Wandel solcher Quartiere im Sinne einer Gentrifizierung. Postulieren Mafaalani et al. (2015, S. 10), dass Segregation in Deutschland politisch gewollt, Mischung hingegen naiv und nicht durchsetzbar sei, so zeigt sich in Zürich ein anderes Bild. Hier verfolgt der Stadtrat das Ziel, eine bessere Durchmischung der Stadtquartiere durch eine aktive Wohnpolitik zu fördern (Stadt Zürich, 2017). Bekräftigt wurde dies von der Wohnbevölkerung durch die Volksabstimmung im Jahr 2011 („Bezahlbare Wohnungen für Zürich“). Betroffen ist auch eine Mehrheit der Schulen im Untersuchungskontext, da nun ehemals sozial und ethnisch segregierte Stadtteile sozialräumlich aufgewertet werden. Diese politisch begleiteten, wenn nicht gesteuerten Gentrifizierungsprozesse bedingen eine erneute Auseinandersetzung der Schule mit dem sich wandelnden sozialen Kontext.

Sind somit Schulen in ,herausfordernder' Lage nicht nur ein geeignetes und vielversprechendes Untersuchungsfeld, sondern stellt sich gerade bei ihnen die Frage nach dem Umgang mit Förderung und Selektion in besonderem Maße, gilt es nun mit Blick auf die Aufgaben und Funktionen der Schule, verschiedene Konzeptionen von Bildungsgerechtigkeit zu umreißen und vom Chancenungleichheitsbegriff abzugrenzen. 
Open Access Dieses Kapitel wird unter der Creative Commons Namensnennung 4.0 International Lizenz (http://creativecommons.org/licenses/by/4.0/deed.de) veröffentlicht, welche die Nutzung, Vervielfältigung, Bearbeitung, Verbreitung und Wiedergabe in jeglichem Medium und Format erlaubt, sofern Sie den/die ursprünglichen Autor(en) und die Quelle ordnungsgemäß nennen, einen Link zur Creative Commons Lizenz beifügen und angeben, ob Änderungen vorgenommen wurden. Die in diesem Kapitel enthaltenen Bilder und sonstiges Drittmaterial unterliegen ebenfalls der genannten Creative Commons Lizenz, sofern sich aus der Abbildungslegende nichts anderes ergibt. Sofern das betreffende Material nicht unter der genannten Creative Commons Lizenz steht und die betreffende Handlung nicht nach gesetzlichen Vorschriften erlaubt ist, ist für die oben aufgeführten Weiterverwendungen des Materials die Einwilligung des jeweiligen Rechteinhabers einzuholen.

(c) (1) 